\title{
Devenir des virus entériques en mer et influence des facteurs environnementaux
}

\author{
Christophe GANTZER $^{a}$, Éric DUBOIS ${ }^{b}$, Jean-Marc CRANCE ${ }^{c}$, Sylviane BILLAUDEL ${ }^{d}$, Helena KOPECKA ${ }^{\mathrm{e}}$, \\ Louis SCHWARTZBROD ${ }^{a}$, Monique POMMEPUY ${ }^{\text {b }}$, Françoise LE GUYADER ${ }^{\text {b }}$ \\ ${ }^{a}$ Laboratoire de virologie, faculté de pharmacie, 5, rue Albert-Lebrun, 54000 Nancy, France \\ ${ }^{\mathrm{b}}$ Laboratoire de microbiologie, Ifremer, BP 21 105, 44311 Nantes cedex 3, France \\ ${ }^{\mathrm{c}}$ Département de biologie cellulaire, CRSSA, 38700 La Tronche, France \\ ${ }^{\mathrm{d}}$ Laboratoire de virologie, faculté de pharmacie, 1, rue Gaston-Veil, 44000 Nantes, France \\ ${ }^{\mathrm{e}}$ Unité de virologie moléculaire, Institut Pasteur, 25, rue du Docteur-Roux, 75724 Paris cedex 15, France
}

(Révisé le 8 juillet 1998, accepté le 8 juillet 1998)

\begin{abstract}
Influence of environmental factors on the survival of enteric viruses in seawater. To improve the knowledge of the survival of enteric viruses in a marine environment, the influence of physico-chemical parameters (temperature, UV, salinity) on the survival of infectious poliovirus 1 and hepatitis A virus (HAV) in seawater was first studied, the influence of suspended solids (SS) on poliovirus adsorption and survival in seawater was then evaluated and the detection of rotavirus genome in environmental samples (shellfish, river water, treated wastewater) was finally investigated. The results show that temperature has a major impact on virus survival in seawater as the time necessary to inactivate $90 \%$ of the virus $\left(\mathrm{T}_{90}\right)$ is 671 days at $4^{\circ} \mathrm{C}$ and only 25 days at $25^{\circ} \mathrm{C}$. Ultraviolet light $\left(42 \mathrm{~mW} \mathrm{~s} \mathrm{~cm}{ }^{-2}\right)$ rapidly inactivates viruses but $\mathrm{HAV}$ is more resistant $\left(\mathrm{T}_{90}=2.6 \mathrm{~min}\right)$ than the poliovirus $1\left(\mathrm{~T}_{90}=1.3 \mathrm{~min}\right)$. By contrast, seawater salinity has no effect on virus survival. In presence of SS, $90 \%$ to $99.9 \%$ of the viruses were adsorbed. This adsorption does not provide any protection for viruses with low SS concentrations $\left(3\right.$ and $15 \mathrm{mg} \mathrm{L}^{-1}$ ) but a slight increase in virus survival was observed with a high SS concentration $\left(500 \mathrm{mg} \mathrm{L}^{-1}\right)$. Finally environmental sample analysis indicated that $20 \%$ shellfish, about $40 \%$ river water and $40 \%$ treated wastewater tested positive for the rotavirus genome. $\odot$ Elsevier, Paris
\end{abstract}

\section{enteric viruses / seawater / survival / environmental factors}

Résumé - Ce travail a pour but de mieux comprendre le devenir des virus en milieu marin. Dans ce cadre, l'influence de certains paramètres physicochimiques (température, UV et salinité) sur le pouvoir infectieux du poliovirus 1 et du virus de l'hépatite A (VHA) en eau de mer a été évalué, puis l'adsorption du poliovirus l sur les matières en suspension (MES) a été étudié, ainsi que les conséquences sur le pouvoir infectieux du virus et enfin le génome des rotavirus a été recherché en milieu naturel (eaux et coquillages). Les résultats montrent que la température est un paramètre important pour la survie des virus puisque le temps nécessaire à inactiver $90 \%$ des virus $\left(\mathrm{T}_{90}\right)$ en eau de mer est de $671 \mathrm{j} \mathrm{à} 4{ }^{\circ} \mathrm{C}$ alors qu'il est seulement de $25 \mathrm{j} \mathrm{à} 25^{\circ} \mathrm{C}$. Le rayonnement ultraviolet $\left(42 \mathrm{~mW} \mathrm{~s} \mathrm{~cm}^{-2}\right)$ testé en pilote expérimental, permet d'obtenir une inactivation rapide du poliovirus $1\left(T_{90}=1,3 \mathrm{~min}\right)$. Le VHA est plus résistant avec un $T_{90}=2,6 \mathrm{~min}$. En revanche la salinité de l'eau de mer n'a pas d'influence sur la survie des virus. En ce qui concerne l'influence des MES, les résultats montrent qu'en présence de 3,15 et $500 \mathrm{mg} \mathrm{L}^{-1}$ de Na-montmorillonite la majorité des virus est adsorbée $(90$ à $99,9 \%)$. Cet état adsorbé ne procure aucune protection aux virus pour les faibles concentrations de MES ( 3 et $15 \mathrm{mg} \mathrm{L}^{-1}$ ) alors qu'une augmentation significative de survie est observée pour les fortes concentrations $\left(500 \mathrm{mg} \mathrm{L}^{-1}\right)$. Enfin, l'analyse d'échantillon de l'environnement a montré que $20 \%$ des fruits de mer, environ $40 \%$ des eaux de rivière et environ $40 \%$ des eaux usées traitées testées contenaient du génome de rotavirus. (C) Elsevier, Paris

virus entériques / eau de mer / survie / facteurs environnementaux 


\section{INTRODUCTION}

Plus de 140 sérotypes de virus entériques sont susceptibles d'être retrouvés dans le milieu marin. Ils y sont apportés soit par les eaux usées urbaines brutes ou épurées, soit par les fleuves. Du fait de leurs propriétés physicochimiques, ces virus pathogènes pour l'homme ne peuvent se multiplier dans le milieu extérieur et ils ne peuvent que persister dans le milieu extérieur [27].

Leur présence constitue un risque de contamination des coquillages qui s'alimentent par filtration de grandes quantités d'eau et concentrent les virus dans leur tube digestif [31]. Des virus ont ainsi été trouvés dans différentes espèces de coquillage $[3,17,22,32]$. Des études épidémiologiques ont démontré le rôle des coquillages comme facteur de risque dans des épidémies de gastroentérites et d'hépatites virales $[6,15,24]$.

La plus grande partie de ces virus se présente dans le milieu hydrique sous forme agrégée ou adsorbée à la surface de particules (argile, sable, sédiment) [20]. L'association des particules virales avec des matières en suspension augmente leur durée de persistance dans l'environnement marin $[4,30]$. Il est vraisemblable que certains facteurs comme la température, la salinité ou le rayonnement UV conditionnent le devenir de ces virus en influençant I'adsorption ou la désorption.

Plusieurs techniques mettent en évidence une contamination virale dans des échantillons cliniques ou de l'environnement. À l'heure actuelle les deux principales sont la culture cellulaire et la biologie moléculaire. L'isolement sur culture cellulaire présente divers inconvénients tels qu'une sensibilité limitée, un délai de résultat pouvant atteindre plusieurs semaines, un coût onéreux ; certains virus ne peuvent être isolés sur culture de cellules. La toxicité de cerlains prélèvernents du milieu extérieur envers les cellules en culture rend encore plus difficile la mise en évidence des particules virales. Cependant la culture cellulaire est la technique de référence car elle seule permet d'affirmer le caractère infectieux des virus. Le développement de la biologie moléculaire permet d'envisager des techniques rapides, spécifiques et très sensibles telles que l'hybridation moléculaire ou l'amplification génique (PCR) pour l'analyse de la contamination du milieu extérieur $[3,14,19,21,33]$. Ces méthodologies mettent en évidence la présence de l'acide nucléique viral mais ne permettent pas de différencier les particules infectieuses des non infectieuses.

Les travaux décrits ici constituent une première tentative pour comprendre le devenir d'un virus en milieu marin littoral. Trois approches ont été réalisées : (1) influence de facteurs physicochimiques (température, salinité et rayonnement ultraviolet) sur le pouvoir infectieux du poliovirus et du virus de l'hépatite A (VHA) ; (2) étude de l'adsorption et de la désorption du poliovirus sur des matières en suspension et des conséquences sur le devenir du pouvoir infectieux du poliovirus ; (3) évaluation in situ de la contamination par des rotavirus en utilisant des techniques de biologie moléculaire.

\section{MATÉRIEL ET MÉTHODES}

\subsection{Souches virales}

Poliovirus : Il s'agit du poliovirus de type 1 souche vaccinale LSC 2ab obtenu par culture sur des cellules MA 104. Virus de l'hépatite A : Le virus utilisé est la souche CF 53 [5] obtenu par culture sur des cellules d'hépato-carcinome humain PLC/PRF/5 à $32^{\circ} \mathrm{C}$.

Rotavirus : une souche de rotavirus simien SAll (souche ATCC VR 899) a été multipliée sur cellules MA 104 et titrée par immunofluorescence indirecte. Des rotavirus isolés de selles d'enfants infectés ont été fournis par le laboratoire hospitalier de Fort-de-France en Martinique.

\subsection{Culture cellulaire et quantification du matériel viral}

Les poliovirus sont quantifiés en culture de cellule MA 104 par la méthode du nombre le plus probable (NPP) en microplaques de 96 cupules. Le calcul du nombre le plus probable NPP de virus contenus dans un inoculum est réalisé en mettant en cuvre le programme informatique de Maul [25]. Les résultats sont exprimés en NPPUC $\mathrm{mL}^{-1}$.

En ce qui concerne le virus de l'hépatite $A$, le titre infectieux est obtenu par détermination de la dose infectieuse $50 \%$ sur cellules PLC/PRF/5 après 5 semaines d'incubation. Le titre infectieux exprimé en DICT 50 est calculé par la méthode de Reed et Muench.

\subsubsection{Analyse statistique}

Pour l'étude des survies, des régressions linéaires sont calculées en tenant compte de tous les points expérimentaux et il est vérifié que les coefficients de corrélation sont significatifs au seuil de $5 \%$. Les équations des régressions sont de type :

$\log N_{t}=a t+\log N_{0}$

(eq 1) 
$N_{t}:$ titre viral au temps $t$ (NPPUC $\mathrm{mL}^{-1}$ )

$N_{0}:$ titre viral initial (NPPUC $\mathrm{mL}^{-1}$ )

$t:$ temps (jours)

$a$ : pente

L'équation 1 permet de calculer $T_{9 n}$, temps au bout duquel le titre viral chute de $90 \%$, correspondant à l'inverse de la pente des droites (a). Les pentes, ou les $\mathrm{T}_{9(1)}$, sont comparés entre eux deux à deux à l'aide d'un test de Student.

\subsection{Analyse par biologie moléculaire}

\subsubsection{Extraction et purification des acides nucléiques}

La lyse des capsides virales est effectuée par action de la protéinase $\mathrm{K}$ en présence d'un mélange détergent sur $410 \mu \mathrm{L}$ de concentrats de coquillages ou d'eaux [22]. Après cxtraction et purification par adsorption sur ccllulose granulaire CC41 [22], le culot d'acide nucléique est repris dans de l'eau stérile traitée au DEPC.

\subsubsection{Amorces et sondes}

Trois amorces et une sondc ont été sćlectionnées à partir de la séquence du gène de la protéine VP7 des rotavirus du groupe $A$ [7].

\subsubsection{RT seminested-PCR.}

L'ARN db des rotavirus est d'abord soumis à une dénaturation préalable à l'aide de l'hydroxyméthylmercure pour sćparcr les dcux brins d'ARN [22]. Les acides nucléiques sont ensuite transcrits en ADN complémentaire (ADNc) à l'aide d'une reverse transcriptase selon les protocoles préconisés par le fabricant (Stratagene).

L'amplification est effectuée selon le protocole préconisé par Perkin Elmer (Cetus) [1, 22]. Pour la détection dans les échantillons naturels une seconde amplification ou seminested PCR est réalisée avec une amorce interne [23].

\subsubsection{Hybridation}

Le marquage des oligosondes est réalisé à l'extrémité 3' selon le protocole DIG oligonucleotide tailing kit (Boehringer Mannheim, France). Après électrophorèse sur gel de polyacrylamide, les acides nucléiques sont transférés à l'aide d'un courant électrique (Fast Blot, Eurogentec) sur une membrane de nylon. L'hybridation et la révélation par chemiluminescence sont réalisées selon le protocole préconisé par le fournisseur (Boehringer Mannheim, France).

\subsubsection{Analyse par RFLP}

Le profil de restriction des séquences amplifiées pour le rotavirus, a été analysé par quatre enzymes de restriction : AluI, RsaI, Sau3AI et TaqI (Eurogentec). Dans le cas où une seule séquence est fortement amplifiée, l'action enzymatique s'effectue directement sur le produil de PCR selon les indications fournies par le fournisseur (Eurogentec). Si l'amplification a été insuffisante et/ou non spécifique, une étape de purification avant une nouvelle amplification est appliquée. La bande spécifique d'acide nucléique amplifiée est purifiée puis analysée par les mĉmes enzymes que celles citées ci-dessus.

\subsection{Analyse en eau de mer synthétique}

\subsubsection{Influence des paramètres physicochimiques sur le devenir du poliovirus et du virus de l'hépatite A.}

L'eau de mer est constituée d'eau désionisée additionnée d'une quantité variable de sels marins synthétiques (Instant Ocean) selon la salinité souhaitée (14, 24, 33). L'eau de mer est ensuite stérilisée par filtration sur filtre Millipore de $0,22 \mu \mathrm{m}$ et conservée à $+4{ }^{\circ} \mathrm{C}$ jusqu'à utilisation. L'hydratation des sels entraînant une différence entre la quantité de produit pesé pour $1 \mathrm{~L}$ d'eau et la salinité, celle-ci a été systématiquement mesurée à l'aide d'un densimètre et d'une table de correspondance densitésalinité.

Influence de la température et de la salinité : les différentes eaux obtenues sont contaminées avec une quantité connue de poliovirus puis réparties, à raison de $100 \mathrm{~mL}$, dans des flacons de $125 \mathrm{~mL}$ bouchés hermétiquement et placés à l'obscurité. Les conditions d'analyse sont :

- la température : $4{ }^{\circ} \mathrm{C}, 19^{\circ} \mathrm{C}$, et $25^{\circ} \mathrm{C}$ avec une salinité de 24.

- la salinité : 14,24 , et 33 . à une température de $25^{\circ} \mathrm{C}$.

Influence du rayonnement ultraviolet : le pilote de désinfection est constitué d'un circuit fermé équipé d'une lampe UV germicide (longueur d'onde $254 \mathrm{~nm}$ ) de $15 \mathrm{~W}$ et dans lequel circule l'eau. Le débit de fonctionnement est de $4 \mathrm{~L} \cdot \mathrm{min}^{\prime}$ et le temps de passage du volume de liquide dans tout le circuit est de 45 s. Pour un débit de $4 \mathrm{~L} \cdot \mathrm{min}^{-1}$, la dose germicide est de $42 \mathrm{~mW} \cdot \mathrm{s} \cdot \mathrm{cm}^{-2}$. L'eau 
de mer de salinité 24 a été contaminée d'une part avec du poliovirus, d'autre part avec du virus de l'hépatite $\mathrm{A}$.

\subsection{2. Étude de l'adsorption et de la désorption du poliovirus}

L'argile utilisée comme modèle de matières en suspension (MES) est la Na-montmorillonite SX.1 purifiée selon le protocole décrit par Gantzer et al. [11].

Pour étudier la cinétique d'adsorption, des lots de cinq flacons sont préparés contenant de l'eau de mer de salinité 33 à des concentrations respectives en Na-montmorillonite de 3,15 et $500 \mathrm{mg} \mathrm{L}^{-1}$ et une quantité connue de poliovirus. Les flacons sont soumis à une agitation magnétique et des échantillons du mélange « eau-virusargile » sont prélevés pour chaque lot après $1,5,10,30$ et $60 \mathrm{~min}$ d'agitation. La quantification est réalisée d'une part sur la suspension $\left(\mathrm{T}_{1}\right)$, d'autre part sur le surnageant de centrifugation de la suspension $\left(20000 \mathrm{~g},+4{ }^{\circ} \mathrm{C}\right.$, $30 \mathrm{~min}$ ) qui représente le titre du virus libre $\left(\mathrm{T}_{2}\right)$. Le pourcentage d'adsorption est ensuite calculé selon la formule :

$\frac{1-\mathrm{T}_{2}}{\mathrm{~T}_{1}} \times 100=\%$ d'adsorption

Pour étudier la désorption, deux liquides d'élution ont été utilisés : un tampon borate-extrait de bœuf $\mathrm{pH} 9$ et de l'eau désionisée. La désorption a été étudiée sur des culots de Na-montmorillonite-poliovirus obtenus avant ou après congélation de ceux-ci dans de l'eau de mer de salinité 33. Les culots Na-montmorillonite-virus sont soumis à une agitation magnétique en présence de l'éluant pendant $30 \mathrm{~min}$. Un aliquote de la suspension obtenue est alors titré pour déterminer le titre viral de la suspension tandis que la suspension est centrifugée ( $30 \mathrm{~min}, 20000 \mathrm{~g},+4^{\circ} \mathrm{C}$ ). La quantité de virus désorbés est alors déterminée par titrage dı surnageant de centrifugation.

Le devenir du poliovirus en présence de MES a été étudié en préparant huit litres d'eau de mer de salinité 33, additionnés de Na-montmorillonite à la dose voulue, contaminés avec une quantité connue de poliovirus puis répartis à raison de $100 \mathrm{~mL}$ dans des flacons en verre de $125 \mathrm{~mL}$ fermés hermétiquement. Le stockage est effectué à l'abri de la lumière et à $25^{\circ} \mathrm{C}$.

\subsection{Analyse in situ}

Les recherches ont été réalisées sur deux types de prélèvements :
- Échantillons d'eaux de surface (eaux de rivières et effluents de stations d'épuration) prélevês, en mars 1993, autour de la baie de Fort-de-France en Martinique (figure 1).

- Coquillages (huîtres et palourdes) collectés dans la région du Morbihan, aux mois de mai, juillet et octobre 1992 et traités comme décrit précédemment par Le Guyader et al. [22].

\section{RÉSULTATS}

\section{1. Étude in vitro}

\subsubsection{Influence des facteurs physicochimiques \\ sur le devenir du poliovirus et du virus de l'hépatite $A$.}

Influence de la température : les différentes températures étaient 4,19 et $25^{\circ} \mathrm{C}$ en eau de mer de salinité 24 contenant du poliovirus, à l'abri de la lumière. Les résultats (tableau I) montrent que, dans les conditions expérimen. tales utilisées, le devenir des virus en eau de mer dépend largement de la température (les trois $\mathrm{T}_{90}$ sont significativement différents au seuil $5 \%$ ). En effet, pour le poliovirus, une augmentation de température de $+4{ }^{\circ} \mathrm{C}$ à $+25^{\circ} \mathrm{C}$ entraîne une chute du $\mathrm{T}_{90}$ d'un facteur 20 environ. L'inactivation de $90 \%$ de la population virale initiale est donc vingt fois plus rapide à $+25^{\circ} \mathrm{C}$ qu'à $+4^{\circ} \mathrm{C}$. Il faut souligner la remarquable stabilité du virus à $+4^{\circ} \mathrm{C}$ puisqu'à cette température le $T_{90}$ est de $671 \mathrm{j}$ alors qu'il est de $25 \mathrm{j}$ à $+25^{\circ} \mathrm{C}$ (tableau $\mathrm{l}$ ).

Influence de la salinité : l'étude a été effectuée avec des échantillons d'eau de mer de salinité 14,24 et 33 contaminée avec du poliovirus. L'analyse des résultats (tableau I) ne montre aucune différence significative entre les trois $\mathrm{T}_{90}$, ce qui prouve que la salinité de l'eau de mer n'a pas d'influence sur le pouvoir infectieux du poliovirus.

Influence du rayonnement ultraviolet : l'étude a été réalisée avec de l'eau de mer de salinité 24. Les essais sont faits en duplicats, d'une part avec le poliovirus, d'autre part avec le virus de l'hépatite A. Il apparaît (tableau I) que l'effet virucide des UV dans une eau de mer non turbide est extrêmement important puisque les deux virus testés sont inactivés en quelques minutes dans les conditions expérimentales. L'analyse des résultats met en évidence une différence significative au seuil de $5 \%$ de sensibilité des deux virus. Le $T_{90}$ est environ deux fois plus élevé pour le VHA, démontrant une plus grande 
Tableau I. Durée de survie $\left(\mathrm{T}_{90}\right)$ des virus en eau de mer stérile.

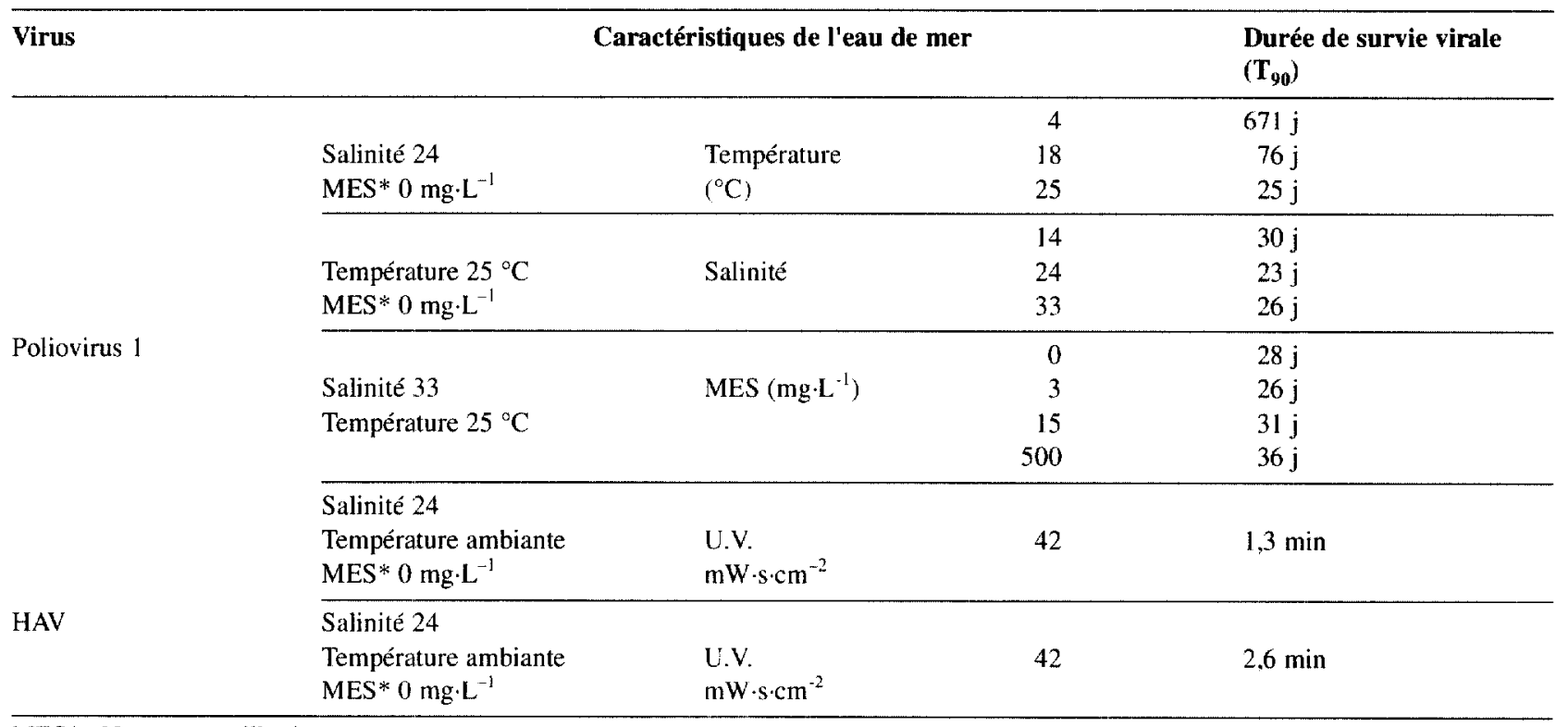

MES* : Na-montmorillonite

résistance de ce virus à l'action du rayonnement ultraviolet.

\subsubsection{Influence des matières en suspension}

Afin d'envisager le devenir du poliovirus en présence de matières en suspension, la cinétique d'adsorption et la désorption du poliovirus ont été étudiées sur de la $\mathrm{Na}$ montmorillonite prise comme modèle de MES.

Dans un premier temps, un dosage de MES dans une eau de mer provenant d'une zone conchylicole atlantique a permis de constater que la concentration en MES variait entre 3 et $15 \mathrm{mg} \mathrm{L}^{-1}$. Compte tenu de ces valeurs, toutes les expérimentations ont été menées avec des concentrations de 3,15 et $500 \mathrm{mg} \mathrm{L}^{-1}$ de Na-montmorillonite.

Les résultats montrent une adsorption virale rapide ( $<30 \mathrm{~min}$ ), quelle que soit la concentration de $\mathrm{Na}$-montmorillonite. En revanche, les pourcentages d'adsorption varient avec la concentration d'argilc. Ils sont respectivement de 71,95 et $>99,9 \%$ pour 3,15 et $500 \mathrm{mg} \mathrm{L}^{-1}$ de $\mathrm{Na}$-montmorillonite après $1 \mathrm{~min}$ d'agitation (tableau $I I$ ).

En présence de 15 et $500 \mathrm{mg} \mathrm{L}^{-1}$, les pourcentages d'adsorption sont maximaux dès la première minute d'agitation. En revanche, pour $3 \mathrm{mg} \mathrm{L}^{-1}$, le pourcentage augmente de $71 \%$ après une minute d'agitation pour atteindre $87 \%$ après $60 \mathrm{~min}$ d'agitation. Cela peut être expliqué par le fait que l'adsorption virale dépendrait de la probabilité de rencontre virus-particule.

La désorption a été étudiée à l'aide de deux liquides d"élution. Lorsque le culot de Na-montmorillonite conte-

Tableau II. Pourcentage d'adsorption du poliovirus en fonction du

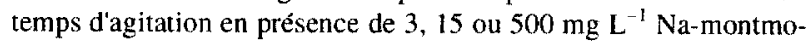
rillonite.

\begin{tabular}{|c|c|c|c|}
\hline \multirow[b]{2}{*}{$\begin{array}{l}\text { Temps } \\
\text { d'agitation (min) }\end{array}$} & \multicolumn{3}{|c|}{$\begin{array}{l}\text { Pourcentage d'adsorption en présence } \\
\text { de Na-montmorillonite }\end{array}$} \\
\hline & $3 \mathrm{mg} \mathrm{L}^{-1}$ & $15 \mathrm{mg} \mathrm{L}^{-1}$ & $500 \mathrm{mg} \mathrm{L}^{-1}$ \\
\hline 1 & $\begin{array}{c}71,0 \\
(61,7 ; 78,0)^{*}\end{array}$ & $\begin{array}{c}95,2 \\
(93,7 ; 96,4)\end{array}$ & $>99,9$ \\
\hline 5 & $\begin{array}{c}\mathbf{8 3 , 6} \\
(78,3: 87,5)\end{array}$ & $\begin{array}{c}\mathbf{9 3 , 7} \\
(92,5 ; 95,2)\end{array}$ & $>99,9$ \\
\hline 10 & $\begin{array}{c}71,0 \\
(61,7 ; 78,0)\end{array}$ & $\begin{array}{c}\mathbf{9 4 , 3} \\
(92,5 ; 95,7)\end{array}$ & $\begin{array}{c}\mathbf{9 9 , 8} \\
(99,7 ; 99,8)\end{array}$ \\
\hline 30 & $\begin{array}{c}\mathbf{6 7 , 3} \\
(56,8 ; 75,2)\end{array}$ & $\begin{array}{c}\mathbf{9 4 , 4} \\
(92,6 ; 95,7)\end{array}$ & $>99,9$ \\
\hline 60 & $\begin{array}{c}\mathbf{8 7 , 2} \\
(83,1 ; 90,3)\end{array}$ & $\begin{array}{c}\mathbf{9 0 , 6} \\
(83,1 ; 90,3)\end{array}$ & $\begin{array}{c}\mathbf{9 8 , 5} \\
(98,0 ; 98,8)\end{array}$ \\
\hline
\end{tabular}

* Intervalle de confiance à $95 \%$ 
nant le virus adsorbé est traité directement par les éluants, le pourcentage de désorption est de $75,6 \%$ avec le tampon borate et de $46,1 \%$ pour l'eau désionisée. Après congélation du culot dans l'eau de mer, les pourcentages sont respectivement de 50,3 et $52,8 \%$.

Cette mise au point a permis d'étudier le devenir d'une souche de poliovirus en présence de $\mathrm{MES}$. Les résultats montrent que, quel que soit le milieu, une chute rapide du titre viral est observée (tableau 1). Ainsi, les $T_{90}$ du témoin, 3,15 et $500 \mathrm{mg} \mathrm{L}^{-1}$ de $\mathrm{Na}$-montmorillonite sont respectivement de $28,26,31$ et $36 \mathrm{j}$. Il s'avère que, dans les conditions expérimentales, les faibles concentrations ( 3 et $15 \mathrm{mg} \mathrm{L}^{-1}$ ) de $\mathrm{Na}$-montmorillonite n'ont pas d'influence sur l'inactivation virale, mais avec une concentration beaucoup plus importante $\left(500 \mathrm{mg} \mathrm{L}^{-1}\right)$, le $\mathrm{T}_{90}$ est légèrement augmenté (environ $8 \mathrm{j}$ ).

\subsection{Analyse en milieu naturel}

\subsubsection{Sensibilité de la recherche de rotavirus par $R T$ - seminested PCR sur des prélèvements de coquillages}

Un lot de coques ne donnant pas de réaction positive par RT seminested PCR a été contaminé artificiellement (injection de la dilution virale dans l'hépatopancréas) par du rotavirus simien de titre connu, la concentration virale dans les coquillages variant de $6 \times 10^{6}$ à 0,6 Foci Fluorescents (F.F.) pour $25 \mathrm{~g}$ de chair. Après élution des virus, extraction des acides nucléiques et purification sur cellulose granulaise CC41, puis mise en cuvre d'une RT seminested PCR, la limite de sensibilité de la technique a été évaluée à $6 \times 10^{3}$ F.F. $25 \mathrm{~g}^{-1}$ de chair de coques.

\subsubsection{Recherche de rotavirus dans les coquillages}

Huit lots d'huîtres et douze lots de palourdes prélevés sur les côtes du Morbihan ont été analysés par RT semi-nested PCR à des fins de détection de l'ARN de rotavirus. Deux échantillons d'huîtres et deux échantillons de palourdes se sont révélés positifs, ce qui traduit la présence de génome de rotavirus dans ces coquillages et donc l'existence d'une contamination plus ou moins récente par des rotavirus.

\subsubsection{Recherche de rotavirus dans des eaux de surface}

Des prélèvements d'eau de rivière et d'effluents de stations d'épuration ont été collectés dans la baie de Fort-deFrance en Martinique (figure 1). L'analyse de ces 19 échantillons montrent la présence d'ARN de rotavirus

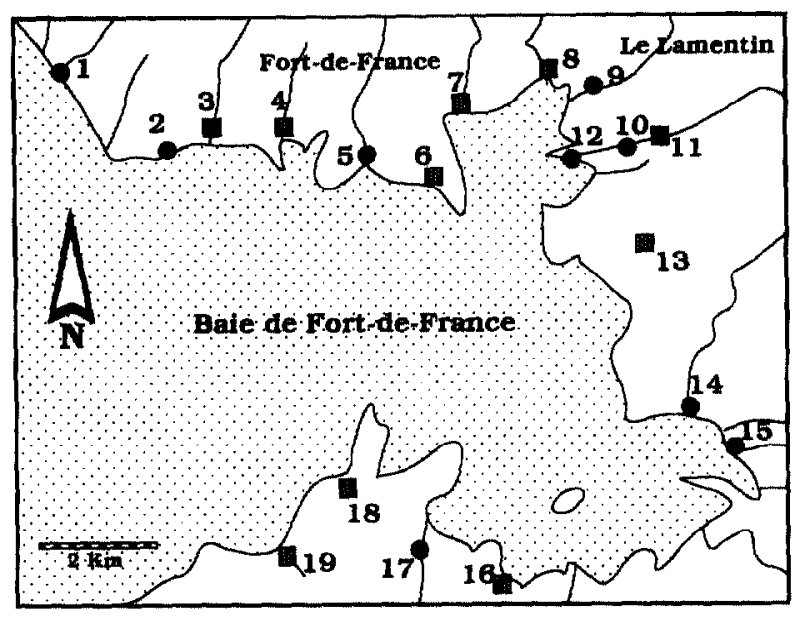

Figure 1. Points de prélèvement en Martinique. : eau de rivière. effluent de station d'épuration.

dans 8 prélèvements, 4 sur 10 pour les effluents de la station d'épuration et 4 sur 9 échantillons d'eau de rivière (tableau III).

\subsubsection{Caractérisation des souches virales par RFLP}

La comparaison des profils de restriction de 6 des 8 séquences virales isolées ( 3 provenant de rivières et 3 d'effluents de station d'épuration) met en évidence des similitudes pour cinq d'entre elles (3 rivières et 2 effluents) avec un profil de souche d'origine humaine. Une séquence virale isolée d'un effluent présente un profil de souche animale.

Ces séquences "environnementales » ont été comparées à celles obtenues à partir de selles d'enfants atteints de rotaviroses (figure 2). Parmi les dix selles étudiées, trois types de profil ont été observés. Aucune similitude n'a pu être mise en évidence entre ces séquences virales isolées de selles et les séquences provenant des eaux.

\section{DISCUSSION}

Les résultats de ce travail apportent quelques éléments de réponse sur le rôle de certains facteurs environnementaux dans le devenir en eau de mer de particules virales entériques telles que le poliovirus. Le devenir des virus en cau de mer dépend largement de la température. Ils sont extrêmement stables à $+4^{\circ} \mathrm{C}\left(\mathrm{T}_{90}=671 \mathrm{j}\right)$ et sont inactivés vingt fois plus vite à 25 qu'à $4{ }^{\circ} \mathrm{C}$. Ces résultats 
Tableau III. Contamination par les rotavirus des eaux de surface de la Martinique.

\begin{tabular}{|c|c|c|c|c|c|c|c|c|c|c|c|c|c|c|c|c|c|c|c|}
\hline $\begin{array}{l}\text { Point } \\
\text { de prélèvement }{ }^{\mathrm{a}}\end{array}$ & 1 & 2 & 3 & 4 & 5 & 6 & 7 & 8 & 9 & 10 & 11 & 12 & 13 & 14 & 15 & 16 & 17 & 18 & 19 \\
\hline $\begin{array}{l}\text { ARNdb } \\
\text { de rotavirus }\end{array}$ & + & + & - & - & + & + & + & + & - & + & - & - & - & - & - & - & - & - & + \\
\hline
\end{tabular}

a : Les numéros correspondent à ceux de la figure 1.

b : Résultats positifs $(+)$ ou négatifs $(-)$ pour la détection d'ARN viral dans les extraits obtenus à partir des prélèvements, par amplification et hybridation moléculaire.

sont en accord avec ceux de Enriquez et al. [9]. La salinité apparaît en revanche comme un paramètre peu important comme l'indiquait Katzenelson [18].

L'influence du rayonnement ultraviolet testée en pilote expérimental est extrêmement importante. L'évolution du poliovirus dans une eau de mer de salinité 24 à $19^{\circ} \mathrm{C}$ montre que le titre viral chute de $99,9 \%$ en 4 min pour une dose de $42 \mathrm{~mW} . \mathrm{s} . \mathrm{cm}^{-2}$. Le virus de l'hćpatitc A cst plus résistant puisqu'une réduction de $99,9 \%$ du titre infecticux est obtenuc au bout de $8 \mathrm{~min}$.

Compte tenu du fait que, en eau de mer, la majorité des virus entériques est associée à des matières en suspension, il était intéressant de déterminer la cinétique d'adsorption et les modalités de désorption du poliovirus
A

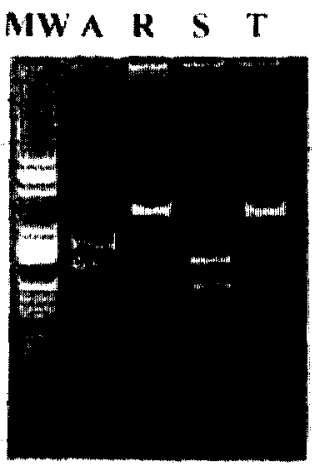

Profil a

\section{A R S T MW}

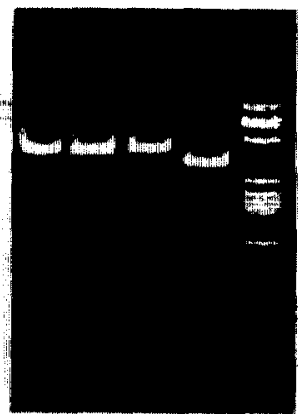

Profil b

\section{B}

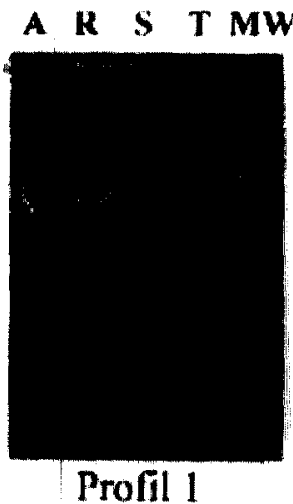

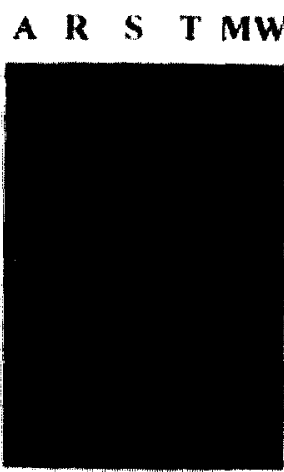

Profil 2

\section{A $\quad R \quad S \quad T \quad M W$}

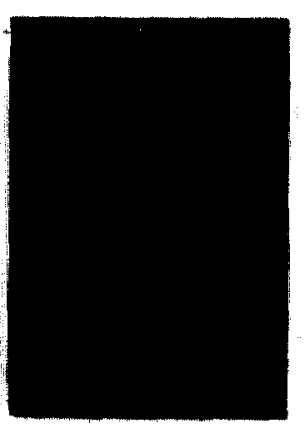

Profil 3

Figure 2. Profils de restriction des séquences de rotavirus amplifiées à partir d'échantillons de l'environnement (A) et de prélèvements cliniques (B). Les séquences amplifiées à partir de prélèvements sont purifiées puis de nouveau amplifiées avant d'être incubées avec les enzymes de restriction AluI (colonnes A), Rsal (colonnes R), Sau3AI (colonnes S) et TaqI (colonnes T). Les produits des réactions de digestion sont séparés par électrophorèse en gel de polyacrylamide. Colonnes $\mathrm{MW}$ : marqueur de taille $\mathrm{V}$ (Boehringer). Les séquences amplifiées à partir des points de prélèvement $1,2,5,7$ et 8 présentaient toutes le profil a. Le profil b correspondait au point 19. Le profil 1 a été obtenu à partir de quatre prélèvements cliniques, le profil deux à partii de deux et le prolïl 3 à partir de quatre. 
sur une argile ( $\mathrm{Na}$-montmorillonite) prise comme modèle de matière en suspension puis d'évaluer si l'état adsorbé a une influence sur la durée de survie des virus en milieu marin. Il est clair que l'adsorption virale est un phénomène très rapide quelle que soit la concentration de $\mathrm{Na}$ montmorillonite mais que, en présence de faibles quantités de Na-montmorillonite, le pourcentage d'adsorption augmente avec le temps pour atteindre un maximum de $87 \%$ après $60 \mathrm{~min}$. Ces résultats sont en accord avec ceux d'Armon et Cabelli [2] qui observent une adsorption de 93 à $99,7 \%$ des bactériophages f2 sur de l'argile en moins de $30 \mathrm{~min}$. Des résultats identiques sont observés avec le poliovirus par Landry et al. [20] et Metcalf et al. [28]. Ces virus sont susceptibles d'être désorbés puisqu'il a été observé une désorption. respectivement de 76 et $46 \%$, en présence d'un tampon borate-extrait de bæuf pH 9 et d'eau désionisée.

L'influence de l'état adsorbé sur la survie du poliovirus a été étudiée dans des conditions de salinité et température déduites des expériences précédentes. Une baisse assez rapide du titre viral est observée pour les faibles concentrations en Na-montmorillonite ( 3 et $15 \mathrm{mg} \mathrm{L}^{-1}$ ) sans différence significative de survie. En revanche, pour une dose de $500 \mathrm{mg} \cdot \mathrm{L}^{-1}$ d'argile, une augmentation légère mais significative de la durée de survie du virus est constatée.

Landry et al. [20], travaillant à des concentrations de $1,2 \mathrm{~g} \mathrm{~L}^{-1}$ de sédiments naturels, avaient observé également une augmentation de $11 \mathrm{j}$ du $T_{90}$ du poliovirus $I$ en eau de mer naturelle à $14^{\circ} \mathrm{C}$ en présence de sédiments par rapport au $T_{90}$ en eau de mer seule.

Il est nécessaire de rappeler que toute cette étude a été réalisée en eau de mer reconstituée, stérile et sans autres matières en suspension que celles ajoutées pour les besoins de l'expérimentation. Cela a permis de connaître l'influence de chaque paramètre. Mais il parait fondamental, dans un deuxième temps, de confronter ces résultats avec ceux qui pourraient être obtenus dans un environnement marin naturel.

La seconde partie du travail a été consacrée à la recherche de rotavirus dans des eaux (eaux de rivière et eaux usées épurées) et des coquillages. Des mises au point méthodologiques notamment dans le choix des amorces pour la détection du génome des rotavirus du groupe A par PCR ont permis d'obtenir une sensibilité comparable à celle décrite dans la littérature $[8,12,13,33]$.

L'analyse des coquillages montre une contamination virale dans $20 \%$ des échantillons analysés. Si les rotavirus sont très résistants [10] et si leur présence avait déjà été rapportée dans des échantillons provenant de l'environnement $[16,26]$, la présence de génome de rotavirus n'avait jusqu'à présent jamais été signalée dans des coquillages.

Les résultats négatifs ne doivent pas être simplement interprétés comme une absence de contamination. En effet, la présence d'inhibiteurs de réaction enzymatique comme le glycogène dans les coquillages peut masquer la présence de virus [3].

Enfin, il faut signaler que, sur les quatre échantillons positifs sur 20 testés, deux présentent une concentration élevée en bactéries entériques alors que deux autres peuvent être considérés comme salubres sur le plan bactériologique, ce qui met en évidence la discordance entre témoins de contamination virale et bactérienne.

En ce qui concerne la présence des rotavirus dans les eaux, la recherche a été effectuée par PCR à partir d'eaux de rivière et d'effluents de stations d'épuration à Fort-deFrance (Martinique). Les pourcentages de positivité sont sensiblement équivalents dans les deux milieux, aux alentours de $40 \%$. Une contamination importante, par les coliformes thermotolérants, a été détectée dans ces échantillons [29]. Il apparaît que l'apport contaminant dans la baie de Fort-de-France provient d'une manière équivalente des rivières et des stations d'épuration. L'observation montre que beaucoup de rejets urbains échappent aux stations d'épuration qui, par ailleurs, présentent des capacités de traitement insuffisantes.

Les séquences, amplifiées à partir des prélèvements positifs, ont été typées par analyse du polymorphisme de restriction. Cette technique, appliquée seulement à une petite partie du génome, permet de distinguer les souches si le profil est différent, mais ne permet pas de conclure à leur identité si les profils sont identiques. Il faudrait dans ce cas analyser une autre partie du génome ou séquencer.

Dans cette étude, l'analyse du polymorphisme de restriction a montré l'existence d'au moins deux souches différentes dans les eaux de la baie de Fort-de-France. Aucune relation n'a été trouvée entre ces souches et celles caractérisées à partir de selles d'enfants malades pendant la même période, mais la comparaison des profils obtenus à partir des prélèvements avec ceux de banques de données suggère une origine humaine.

En termes de prospective ces travaux devraient être poursuivis, en particulier pour étudier simultanément et comparativement, dans les eaux et dans les coquillages, le devenir du pouvoir infectieux des virus entériques, et celui du génome viral. 


\section{RÉFÉRENCES}

[1] Apaire-Machais V., Ferre-Aubineau V., Colona F., Dubois F., Ponge A., Billaudel S., Development of RT semi-nested PCR for detection of hepatitis A virus in stool in epidemic conditions, Mol. Cell. Probes 8 (1994) 101-108.

[2] Armon R., Cabelli V.J., Phage f2 desorption from clay in estuarine water using non ionic detergents, beef extract and chaotropic agents, Can. J. Microbiol. 34 (1988) 1022-1024.

[3] Atmar R.L., Neill F.H., Romalde L., Le Guyader F., Woodley C.M., Metcalf T.G., Estes M.K., Detection of Norwalk virus and hepatitis A virus in shellfish tissues with the PCR. Appl. Environ. Microbiol. 61 (1995) 3014-3018.

[4] Chung H., Sobsey M.D., Comparative survival of indicator viruses and enteric viruses in seawater and sediment, Wat. Sci. Technol. 27 (1993) 425-428.

[5] Crance J.M., Passagot J., Biziagos E., Deloince R., Continuous production of hepatitis A virus in PLC/PRF/5 cell cultures : use of antigen for serology. J. Virol. Meth. 18 (1987) 193203

[6] Desenclos J.C.A., Klentz K.C., Wilder M.H., Naiman O.V., Margalis H.S., Gunn R.A., A multistate outbreak of hepatitis A caused by the consumption of raw oysters, Am. J. Public Health 81 (1991) 1268-1272.

[7] Dubois E.. Contamination de l'environnement littoral par les rotavirus du groupe A, Doct. Univ. Rennes-1 (1995) 143 p.

[8] Eiden J.J., Wilde J., Firoozmand F, Yolken R., Detection of animal and human group $\mathrm{B}$ rotaviruses in fecal specimens by polymerase chain reaction, J. Clin. Microbiol. 29 (1991) 539543

[9] Enriquez C.E., Hurst C.J., Gerba C.P., Survival of the enteric Adenoviruses 40 and 41 in tap, sea and wastewater, Wat. Res. 29 (1995) 2548--2553.

[10] Estes M.K., Rotaviruses and their replication, in : Fields B.N. (éd.), Virology, 3rd ed. Raven Press, New York, 2 (1996) $1625-1655$.

[1I] Gantzer C., Quignon F., Schwartzbrod L., Poliovirus 1 adsorption onto and desorption from Montmorillonite in seawater. Survival of the adsorbed virus, Environ. Tech. 15 (1994) 271278

[12] Gentsch J.R., Glass R.I., Woods P., Gouvea V., Gorziglia M., Flores J., Das B.K., Bhan M.K., Identification of group A rotavirus gene 4 types by polymerase chain reaction, J. Clin. Microbiol. 30 (1992) 1365-1373.

[13] Gouvea V., Allen J.R., Glass R.I., Fang Z.Y., Bremont M., Cohen J., McCrae M.A., Saif L.J., Sinarachatanant P., Caul E.O., Detection of group B and C rotaviruses by polymerase chain reaction, J. Clin. Microbiol. 29 (1991) 519-523.

[14] Gouvea V., Ramirez C., Li B., Santos N., Saif L. Clark H.F., Hoshino Y., Restriction endonuclease analysis of the VP7 gene of human and animal rotaviruses. J. Clin. Microbiol. 31 (1993) $917-923$.
[15] Halliday M.L., Kang L.Y., Zhou T.K., Hu M.D., Pan Q.C., Fu T.Y., Huang Y.S., Hu S.L., An epidemic of hepatitis A attribuable to the ingestion of raw clams in Shanghai China, J. Infect. Dis. 164 (1991) 852-859.

[16] Hejkal T.W., Smith E.M., Gerba C.P., Seasonal occurence of rotavirus in sewage, Appl. Environ. Microbiol. 47 (1984) 588590.

[17] Jofre J., Lucena F., Gajardo R., Bosch A., Detection of human enteric viruses in mussels (Mytilus edulis), in: Gerba C.P., Farrah S. (éd.), Contamination of the environment by viruses and method of control, Lewis Ann Arbor, 1989, USA.

[18] Katzenelson E., Survival of viruses, in : Berg G. (éd.), Indicators of viruses in water and food, Ann Arbor Science, Ann Arbor, MI, 1978, 39-50.

[19] Kopecka H., Dubrou S., Prevot J., Marechal J., Lopez-Pila J.M., Detection of naturally occuring enteroviruses in waters by reverse transcription, polymerase chain reaction, and hybridization, Appl. Environ. Microbiol. 59 (1993) 1213-1219.

[20] Landry E.F., Vaughn J.M., Vicale T.J., Mann R., Accumulation of sediment associated viruses in shellfish, Appl. Environ. Microbiol. 46 (1983) 673-682.

[21] Le Guyader F., Dincher M.L., Menard D., Schwartzbrod L., Pommepuy M., Comparative study of the behavior of poliovirus in sterile seawater using RT-PCR and cell culture, Mar. Poll. Bull. 28 (1994) 723-726.

[22] Le Guyader F., Dubois E., Menard D., Pommepuy M., Detection of hepatitis A virus, Rotavirus, and enterovirus in naturally contaminated shellfish and sediment by reverse transcription-seminested PCR, Appl. Environ. Microbiol. 60 (1994) 3665-3671.

[23] Le Guyader F., Menard D., Pommepuy M., Kopecka H., Use of RT seminested PCR to assess viral contamination in Carribbean rivers (Martinique), Wat. Sci. Tech. 31 (1995) 391-394.

[24] Le Guyader F., Miossec L., Atmar R.L., Neill F.H., Dubois E., Haugarreau L., Estes M.K., Pommepuy M., An SRSV outbreak linked to shellfish consumption in France, Abstr. P5-2. p.167, in : American Society for Virology, 15th anmual meeting, Scientific program and abstracts (1996).

[25] Maul A., Aspects statistiques des méthodes de quantification en virologie, in : Schwartzbrod L. (éd.), Virologie des milieux hydriques, Tec et Doc, Paris, 1991, p. 143-173.

[26] Mehnert D.U., Stewien K.E., Detection and distribution of rotavirus in raw sewage and creeks in Sao Paulo, Brazil, Appl. Environ. Microbiol. 59 (1993) 140-143.

[27] Melnick J.L., Gerba C.P., Wallis C., Viruses in water, Bull World Health Organization 56 (1978) 499-508

[28] Metcalf T.G., Rao V.C., Melnick J.L., Solid associated viruses in a polluted estuary, Monog. Virol. 15 (1984) 97-110.

[29] Pommepuy M., Derrien A., Le Guyader F., Menard D., Caprais M.C., Dubois E., Dupray E., Gourmeton M., Micro- 
bial water quality in a Carribbean island (Martinique), Coastal and Estuarine Studies 51 (1996) 284-297.

[30] Rao V.C., Seidel K.M., Goyal S.M., Metcalf T.G., Melnick J.L., Isolation of enteroviruses from water, suspended solids, and sediments from Galveston Bay: survival of poliovirus and rotavirus adsorbed to sediments, A.ppl. Environ. Microbiol. 48 (1984) 404-409.

[31] Romalde J.L., Estes M.K., Szucs G., Atmar R.L., Woodley C.M., Metcalf T.G., in situ detection of hepatitis A virus in cell cultures and shellfish tissues, Appl. Environ. Microbiol. 60 (1994) 1921-1926.

[32] Wait D.A., Hackney C.A., Carrick R.J., Lovelage G., Sobsey M.D., Enteric bacterial and viral pathogens and indicator bacteria in hard shell clams, J. Food Protect. 46 (1983) 493-498.

[33] Wilde J., Eiden J., Yolen R., Removal of inhibitory substances from human fecal specimens for detection of group A rotaviruses by reverse transcriptase and polymerase chain reactions, J. Clin. Microbiol. 28 (1990) 1300-1307. 